Research Article

\title{
Inverse Eigenvalue Problems for Singular Rank One Perturbations of a Sturm-Liouville Operator
}

\author{
Xuewen Wu \\ School of Mathematics and Statistics, Northwestern Polytechnical University, Xi'an 710072, China \\ Correspondence should be addressed to Xuewen Wu; xuewenwu1991@163.com
}

Received 11 April 2021; Accepted 20 July 2021; Published 4 August 2021

Academic Editor: Pavel Kurasov

Copyright (C) 2021 Xuewen Wu. This is an open access article distributed under the Creative Commons Attribution License, which permits unrestricted use, distribution, and reproduction in any medium, provided the original work is properly cited.

This paper is concerned with the inverse eigenvalue problem for singular rank one perturbations of a Sturm-Liouville operator. We determine uniquely the potential function from the spectra of the Sturm-Liouville operator and its rank one perturbations.

\section{Introduction}

Consider the boundary problem

$$
\tilde{L} y:=-y^{\prime \prime}+q(x) y+\alpha \delta\left(x-x_{0}\right) y\left(x_{0}\right)=\lambda y
$$

on $(0,1)$ with

$$
y^{\prime}(0)=y(1)=0
$$

where $q(x) \in L^{2}(0,1)$ is real valued, $\alpha \in \mathbb{R} \backslash\{0\}, x_{0} \in(0,1)$, and $\delta(x)$ are the Dirac delta function. It is well known [1] that the operator $\tilde{L}$ is a self-adjoint operator in $L^{2}(0,1)$, which is a singular rank one perturbation of the SturmLiouville operator $L y:=-y^{\prime \prime}+q(x) y$.

The goal of this paper is to deal with the inverse problem of recovering the potential $q(x)$ in (1) from the spectra of $L$ and $\tilde{L}$, by applying the method in [1] and the perturbation theory for linear operators [2]. Note that in [3], the boundary problem

$$
-y^{\prime \prime}+q(x) y=\lambda y
$$

with (2) and the discontinuous conditions

$$
y\left(x_{0}+0\right)=y\left(x_{0}-0\right)=y\left(x_{0}\right)
$$

$$
y^{\prime}\left(x_{0}+0\right)=y^{\prime}\left(x_{0}-0\right)+\alpha y\left(x_{0}\right)
$$

can be regarded as the problem (1)-(2). The research of this paper can be a variation of Borg's two-spectra theorem [4] for the second spectrum that is obtained by attaching the interface conditions (4) and (5) to the problem (3) and (2). Our immediate motivation is a recent research of del Rio and Kudryavtsev [5,6], who considered the inverse problem for Jacobi matrices and recovered the original matrix from the spectra of it and its interior mass-spring perturbation; they indicated that the uniqueness for the inverse problem does not remain valid in general; however, under certain conditions, there exists at most a finite number of matrices corresponding to the two spectra.

Such operator $\tilde{L}$ appears not only in electronics but in other areas such as the theory of diffusion processes, see the related references in $[7,8]$. Some spectral and inverse spectral problems for the Sturm-Liouville operator with rank one perturbations have been investigated in [1, 3, 9-20]. In particular, Albeverio, Hryniv, and Nizhnik [3] considered the inverse eigenvalue problem for the Sturm-Liouville operator with the point potential $v(x)(y, \delta)_{L^{2}}$ and the perturbation $\delta$ $\left(x-x_{0}\right)(y, v)_{L^{2}}$ where $v(x) \in L^{2}(0,1)$ and $x_{0}=1$. Later, Nizhnik [16] continued the problem with $x_{0} \in(0,1)$. However, we consider the inverse problem for the operator (1) with the potential $q(x) \in L^{2}(0,1)$ and the perturbation $\delta\left(x-x_{0}\right)$ $(y, \delta)_{L^{2}}$. And the potential may not be determined uniquely just by the spectra; so, we employ the addition information. Moreover, the approach we use can also solve the problem 
with the perturbation $c(x)(y, c)_{L^{2}}, c(x) \in L^{2}(0,1)$ in the research [18]. In the paper, we establish the expression of the characteristic function of $\tilde{L}$ which provides a necessary preliminary for treating with its inverse eigenvalue problem. The approach we use to prove our results can convert the problem (1) and (2) into three spectra inverse problem in $[21,22]$. Actually, the spectra of $L$ and $\tilde{L}$ may not determine the potential uniquely (see Remark below for details). The key difficulty encountered is to identify the eigenvalues of two Sturm-Liouville problems defined on $\left[0, x_{0}\right]$ and $\left[x_{0}, 1\right]$ from the knowledge of the spectra of $L$ and $\tilde{L}$, for which we have to employ addition information of the number of zeros of the eigenfunctions, and the condition two spectra are disjoint.

The main result asserts that, if the spectra of $L$ and $\tilde{L}$ are disjoint, the potential $q(x)$ can be determined uniquely by the spectra of $L$ and $\tilde{L}$ and the numbers of zeros, contained in ( $\left.0, x_{0}\right)$, of all eigenfunctions of $L$. We will state and prove it in the next section.

\section{The Main Theorem and Proof}

We describe some preliminaries which will be needed subsequently, due to [1]. One defines the scale of spaces $H_{ \pm 1}(L)$ associated to $L$ as follows. The space $H_{+1}(L)$ is $D\left(L^{1 / 2}\right)$ with the norm

$$
\|\varphi\|_{H_{+1}}=\left\|(L+1)^{1 / 2} \varphi\right\|_{L^{2}(0,1)}
$$

in which $H_{+1}$ is easily seen to be complete. For $H_{-1}(L)$, take $L^{2}(0,1)$ with the norm given by

$$
\|\varphi\|_{H_{-1}}=\left\|(L+1)^{-1 / 2} \varphi\right\|_{L^{2}(0,1)}
$$

and complete it. Note that $H_{+1}(L)$ and $H_{-1}(L)$ are dual in such a way that $\varphi \in H_{-1}$ is associated to the function $y \in$ $H_{+1}$ given by $\int_{0}^{1} \varphi(x) y(x) d x$. A Sobolev estimate shows that, for any $y \in H_{+1}(L)$,

$$
\left(\int_{0}^{1} y(x) \delta\left(x-x_{0}\right) d x\right)^{2}=\left|y\left(x_{0}\right)\right|^{2} \leq c(y,(L+1) y)<\infty
$$

that is, $\delta\left(x-x_{0}\right)$ lies in $H_{-1}(L)$. Hence, by Section 2 in ([1], p. 115) Simon, one can find a spectral measure $d \mu_{L}$ such that

$$
F(z)=\int_{0}^{1}\left(\delta\left(x-x_{0}\right),(L-z)^{-1} \delta\left(x-x_{0}\right)\right) d x \equiv \int_{0}^{1} \frac{d \mu_{L}(x)}{x-z} .
$$

By (I.15) and (I.16) in ([1], p. 116), it follows that

$$
\operatorname{Tr}\left[(L-z)^{-1}-(\tilde{L}-z)^{-1}\right]=\frac{\alpha(d F(z) / d z)}{1+\alpha F(z)} .
$$

The following preliminaries are due to ([2], p. 245-250). The multiplicity index for $\alpha F(z)+1$ is given by $v(\zeta ; \alpha F(z)+1)=\left\{\begin{array}{l}k, \text { if } \zeta \text { is a zero of } \alpha F(z)+1 \text { of order } k, \\ -k, \text { if } \zeta \text { is a pole of } \alpha F(z)+1 \text { of order } k \\ 0, \text { for all other } \zeta \in \mathbb{C}\end{array}\right.$

The multiplicity function for a closed operator $T$ is defined by

$\tilde{v}(\zeta ; T)=\left\{\begin{array}{l}0, \text { if } \zeta \text { belongs to the resolvent set, } \\ m, \text { if } \zeta \text { is a neigenvalue of } T \text { with multiplicity } m \\ +\infty, \text { for all other } \zeta \in \mathbb{C} .\end{array}\right.$

Let $\lambda_{n}$ be the eigenvalue of $L, n \geq 0$. Then,

$$
\tilde{v}(\zeta ; L)=\left\{\begin{array}{l}
1, \zeta \in\left\{\lambda_{n}\right\}_{n=0}^{\infty} \\
0, \mathbb{C} \backslash\left\{\lambda_{n}\right\}_{n=0}^{\infty}
\end{array}\right.
$$

Using the same way as the proof of the $\mathrm{W}$-A formulas in ([2], p. 248), by (10), we deduce

$$
\begin{aligned}
\tilde{v}(\zeta ; \tilde{L})= & v(\zeta ; \alpha F(z)+1)+\tilde{v}(\zeta ; L) \\
& =\left\{\begin{array}{l}
1+v(\zeta ; \alpha F(z)+1), \zeta \in\left\{\lambda_{n}\right\}_{n=0}^{\infty}, \\
v(\zeta ; \alpha F(z)+1), \zeta \in \mathbb{C} \backslash\left\{\lambda_{n}\right\}_{n=0}^{\infty} .
\end{array}\right.
\end{aligned}
$$

In the following lemma, we give the spectrum and a characteristic function of $\tilde{L}$.

Lemma 1. The spectrum of $\tilde{L}$ consists of real eigenvalues. The characteristic function of $\tilde{L}$ is

$$
\tilde{\Delta}(\lambda)=\Delta(\lambda)(\alpha F(\lambda+i 0)+1)
$$

where $\Delta(\lambda)$ is the characteristic function of $L$.

Proof. By (9),

$$
F(z)=G\left(x_{0}, x_{0}, z\right)
$$

where $G(x, y, z)$ is Green's function for $L$. From ([23], p. 15, p.29), $G\left(x_{0}, x_{0}, z\right)$ is meromorphic with simple poles in the points $z=\lambda_{n_{i}}$. Then, for $\lambda \in \mathbb{R} \backslash\left\{\lambda_{n_{i}}\right\}_{i=0}^{\infty}$, it follows

$$
F(\lambda+i 0)=F(\lambda)
$$

$$
\frac{d F(\lambda+i 0)}{d \lambda}=\int \frac{d \mu_{L}(y)}{(y-\lambda)^{2}}=\frac{d G\left(x_{0}, x_{0}, \lambda\right)}{d \lambda}<\infty .
$$

Notice that the spectrum of $L$ consists of simple real eigenvalues. Combining with Theorem I.6 in [1], we see 


$$
\operatorname{Im} F(\lambda+i 0)=\left\{\begin{array}{l}
\infty, \lambda \in\left\{\lambda_{n}\right\}_{n=0}^{\infty}, \\
0, \lambda \in \mathbb{R} \backslash\left\{\lambda_{n}\right\}_{n=0}^{\infty},
\end{array}\right.
$$

Thus, by Theorem II.2 in [1] and (18), the spectrum of $\tilde{L}$ consists of real eigenvalues, denoted by $\left\{\mu_{n}\right\}_{n=0}^{\infty}$.

By (14), each zero of $\alpha F(z)+1$ is an eigenvalue of $\tilde{L}$. Hence, the zeros of $\alpha F(z)+1$ are real and consist of the zeros of $\alpha F(\lambda+i 0)+1$. Let $\left\{a_{i}\right\}_{i=0}^{\infty}$ be the zeros set of $\alpha F(\lambda+i 0)$ +1 . Then, we have the multiplicity function

$$
v(\zeta ; \alpha F(z)+1)=\left\{\begin{array}{l}
1, \zeta \in\left\{a_{i}\right\}_{i=0}^{\infty}, \\
-1, \zeta \in\left\{\lambda_{n_{i}}\right\}_{i=0}^{\infty}, \\
0, \text { for other } \zeta \in \mathbb{C} .
\end{array}\right.
$$

Using (14), it follows

$$
\tilde{v}(\zeta ; \tilde{L})=\left\{\begin{array}{l}
2, \zeta \in\left\{a_{i}\right\}_{i=0}^{\infty} \cap\left\{\lambda_{n}\right\}_{n=0}^{\infty}, \\
1, \zeta \in\left\{a_{i}\right\}_{i=0}^{\infty} \backslash\left(\left\{a_{i}\right\}_{i=0}^{\infty} \cap\left\{\lambda_{n}\right\}_{n=0}^{\infty}\right), \\
1, \zeta \in\left\{\lambda_{n}\right\}_{n=0}^{\infty} \backslash\left(\left\{a_{i}\right\}_{i=0}^{\infty} \cap\left\{\lambda_{n}\right\}_{n=0}^{\infty} \cup\left\{\lambda_{n_{i}}\right\}_{i=0}^{\infty}\right), \\
0, \text { for other } \zeta \in \mathbb{C} .
\end{array}\right.
$$

Hence, by the definition of $\tilde{v}(\zeta ; \tilde{L})$, we have

$$
\left\{\mu_{n}\right\}_{n=0}^{\infty}=\left\{a_{i}\right\}_{i=0}^{\infty} \cup\left(\left\{\lambda_{n}\right\}_{n=0}^{\infty} \backslash\left\{\lambda_{n_{i}}\right\}_{i=0}^{\infty}\right) .
$$

Now, we prove that, for $x_{0} \in \mathbb{R}, \tilde{\Delta}\left(x_{0}\right)=0$, if and only if $x_{0} \in\left\{\mu_{n}\right\}_{n=0}^{\infty}$. In fact, if $x_{0} \in\left\{\mu_{n}\right\}_{n=0}^{\infty}$, it is easily seen that $\tilde{\Delta}\left(x_{0}\right)=0$. We just prove that if $\tilde{\Delta}\left(x_{0}\right)=0$, then $x_{0} \in$ $\left\{\mu_{n}\right\}_{n=0}^{\infty}$. Suppose that $\tilde{\Delta}\left(x_{0}\right)=0$ and $x_{0} \notin\left\{\mu_{n}\right\}_{n=0}^{\infty}$, then $x_{0} \in\left\{\lambda_{n_{i}}\right\}_{i=0}^{\infty}$, i.e., $x_{0}$ is a pole of $F(z)$. By Theorem 1.1.2 in [23], all zeros of $\Delta(\lambda)$ are simple; so,

$$
\frac{d \Delta}{d \lambda}\left(x_{0}\right) \neq 0 .
$$

By (15), (16), and ([23], p. 15), $\tilde{\Delta}(\lambda)$ is derivable; thus,

$$
\frac{d \tilde{\Delta}}{d \lambda}\left(x_{0}\right)=\frac{d \Delta}{d \lambda}\left(x_{0}\right)\left(\alpha F\left(x_{0}+i 0\right)+1\right)<\infty,
$$

which is contradictory to that $x_{0}$ which is the pole of $F(z)$. Then, $x_{0} \in\left\{\mu_{n}\right\}_{n=0}^{\infty}$.

For

$$
\frac{d^{2} \tilde{\Delta}}{d \lambda^{2}}\left(\mu_{n}\right) \neq 0
$$

we also need to prove that $x_{0}$ is an eigenvalue of $\tilde{L}$ of multiplicity 2 if and only if $x_{0}$ is a zero of $F(\lambda)$ of multiplicity 2 . Actually, if $x_{0}$ is an eigenvalue of $\tilde{L}$ of multiplicity 2, then by (21), there is $x_{0} \in\left\{a_{i}\right\}_{i=0}^{\infty} \cap\left\{\lambda_{n}\right\}_{n=0}^{\infty}$, and it is easily to get

$$
\tilde{\Delta}\left(x_{0}\right)=\frac{d \tilde{\Delta}}{d x}\left(x_{0}\right)=0 .
$$

Now, we suppose that $x_{0}$ is a zero of $F(\lambda)$ of multiplicity 2, i.e., (26) holds. By $\tilde{\Delta}\left(x_{0}\right)=0$, there is $\Delta\left(x_{0}\right)=0$ or $\alpha F\left(x_{0}+\right.$ i0) $+1=0$. Combining with

$$
\begin{aligned}
\frac{d \tilde{\Delta}}{d \lambda}\left(x_{0}\right)= & \frac{d \Delta}{d \lambda}\left(x_{0}\right)\left(\alpha F\left(x_{0}+i 0\right)+1\right) \\
& +\Delta\left(x_{0}\right) \frac{d}{d \lambda}\left(\alpha F\left(x_{0}+i 0\right)+1\right)=0,
\end{aligned}
$$

there is $\Delta\left(x_{0}\right)=0$ and $\alpha F\left(x_{0}+i 0\right)+1=0$ which mean $x_{0}$ $\in\left\{a_{i}\right\}_{i=0}^{\infty} \cap\left\{\lambda_{n}\right\}_{n=0}^{\infty}$. Then, $x_{0}$ is an eigenvalue of $\tilde{L}$ of multiplicity 2 . The proof is complete.

The main result in this paper is as follows.

Theorem 2. Let $\left\{\lambda_{n}\right\}_{n=0}^{\infty}$ and $\left\{\mu_{n}\right\}_{n=0}^{\infty}$ be the spectrum of $L$ and $\tilde{L}$, respectively. If $\left\{\lambda_{n}\right\}_{n=0}^{\infty} \cap\left\{\mu_{n}\right\}_{n=0}^{\infty}=\varnothing$, the potential $q(x)$ can be determined uniquely by $\left\{\lambda_{n}\right\}_{n=0}^{\infty},\left\{\mu_{n}\right\}_{n=0}^{\infty}$ and the numbers of zeros, contained in $\left(0, x_{0}\right)$, of all eigenfunctions of $L$.

Proof. By Theorem 1.1.4 in [23], the characteristic function of $L$ can be calculated by

$$
\Delta(\lambda)=\prod_{n=0}^{\infty} \frac{\lambda_{n}}{((\pi / 2)+n \pi)^{2}}\left(1-\frac{\lambda}{\lambda_{n}}\right) .
$$

In the same way of the proof of Theorem 1.1.4 in [23], by Hadamard's factorization theorem [24], the characteristic function of $\tilde{L}$ can be calculated by

$$
\tilde{\Delta}(\lambda)=\prod_{n=0}^{\infty} \frac{\mu_{n}}{((\pi / 2)+n \pi)^{2}}\left(1-\frac{\lambda}{\mu_{n}}\right) .
$$

From the given spectra $\left\{\lambda_{n}, \mu_{n}\right\}_{n=0}^{\infty}$, we can determine the function $F(\lambda+i 0)$ by

$$
F(\lambda+i 0)=\frac{1}{\alpha}\left(\frac{\tilde{\Delta}(\lambda)}{\Delta(\lambda)}-1\right) .
$$

From (16), ([23], p. 15) and (2) in [23],

$$
F(\lambda+i 0)=-\frac{\varphi\left(x_{0}, \lambda\right) \psi\left(x_{0}, \lambda\right)}{\Delta(\lambda)},
$$

where $\varphi(x, \lambda)$ and $\psi(x, \lambda)$ are the solutions of (3) under the initial conditions $\varphi(0, \lambda)=1, \varphi^{\prime}(0, \lambda)=0$ and $\psi(1, \lambda)=0, \psi^{\prime}$ $(1, \lambda)=1$. Then, we get the zeros of $\varphi\left(x_{0}, \lambda\right) \psi\left(x_{0}, \lambda\right)$, denoted by $\left\{\gamma_{n}\right\}_{n=0}^{\infty}$. It should be noted that 


$$
\left\{\gamma_{n}\right\}_{n=0}^{\infty}:=\left\{\gamma_{n}^{-}\right\}_{n=0}^{\infty} \cup\left\{\gamma_{n}^{+}\right\}_{n=0}^{\infty},
$$

where $\left\{\gamma_{n}^{-}\right\}_{n=0}^{\infty}$ and $\left\{\gamma_{n}^{+}\right\}_{n=0}^{\infty}$ are the spectra of the following problems

$$
\begin{aligned}
& \left\{\begin{array}{l}
L^{-} y=-y^{\prime \prime}+q(x) y \text { on }\left(0, x_{0}\right), \\
y^{\prime}(0)=y\left(x_{0}\right)=0
\end{array}\right. \\
& \left\{\begin{array}{l}
L^{+} y=-y^{\prime \prime}+q(x) y \text { on }\left(x_{0}, 1\right), \\
y\left(x_{0}\right)=y(1)=0
\end{array}\right.
\end{aligned}
$$

respectively.

If $\left\{\lambda_{n}\right\}_{n=0}^{\infty} \cap\left\{\mu_{n}\right\}_{n=0}^{\infty}=\varnothing, \lambda_{n}$ is the pole of $F(\lambda+i 0)$, and $\gamma_{n}$ is the zero of $F(\lambda+i 0)$. Using (18), it implies that $F(\lambda+$ i0) is monotonically increasing on $\mathbb{R} \backslash\left\{\lambda_{n}\right\}_{n=0}^{\infty}$. By (16) and ([23], p. 29, [30]),

$$
\begin{aligned}
& \lim _{\lambda \longrightarrow \lambda_{n}^{-}} F(\lambda+i 0)=+\infty, \\
& \lim _{\lambda \longrightarrow \lambda_{n}^{+}} F(\lambda+i 0)=-\infty, \\
& \lim _{\lambda \longrightarrow-\infty} F(\lambda+i 0)=0 .
\end{aligned}
$$

Consequently, there is no zero of $F(\lambda+i 0)$ in $\left(-\infty, \lambda_{0}\right)$ and exactly one zero in $\left(\lambda_{n}, \lambda_{n+1}\right)$. That means

$$
\lambda_{n}<\gamma_{n}<\lambda_{n+1} .
$$

We identify $\left\{\gamma_{n}^{-}\right\}_{n=0}^{\infty}$ and $\left\{\gamma_{n}^{+}\right\}_{n=0}^{\infty}$ from $\left\{\gamma_{n}\right\}_{n=0}^{\infty}$ by virtue of the number of the zeros of the eigenfunction contained in $\left(0, x_{0}\right)$. As is known $([25]$, p. 15$)$ that the eigenfunction $\varphi(x$, $\left.\lambda_{i}\right)$ has exactly $i$ zeros in $(0,1)$, we suppose that the eigenfunction $\varphi\left(x, \lambda_{i}\right)$ has $m(m \leq i)$ zeros in $\left(0, x_{0}\right)$. By comparison theorem in ([25], p14), there are not fewer than $m$ zeros of the eigenfunction $\varphi\left(x, \lambda_{i+1}\right)$ contained in $\left(0, x_{0}\right)$. From Lemma 1.3.1 in [25], the roots of $\varphi(x, \lambda)$ depend continuously on $\lambda$. Combining with Corollary 1.3.2 in [25], $\varphi\left(x, \lambda_{i+1}\right)$ has $m+1$ zeros in $\left(0, x_{0}\right)$ only if there exists $\gamma_{p}^{-} \in\left(\lambda_{i}, \lambda_{i+1}\right)$ such that $\varphi$ $\left(x_{0}, \gamma_{p}^{-}\right)=0$. Therefore, if $\varphi\left(x, \lambda_{i+1}\right)$ has $m+1$ zeros in $(0$, $\left.x_{0}\right)$, then $\gamma_{i} \in\left\{\gamma_{n}^{-}\right\}_{n=0}^{\infty}$; otherwise, $\gamma_{i} \in\left\{\gamma_{n}^{+}\right\}_{n=0}^{\infty}$. Then, we obtain $\left\{\gamma_{n}^{+}\right\}_{n=0}^{\infty}$ and $\left\{\gamma_{n}^{-}\right\}_{n=0}^{\infty}$ from $\left\{\gamma_{n}\right\}_{n=0}^{\infty}$ with the information of the number of zeros of the eigenfunctions $\varphi\left(x, \lambda_{i}\right)$ contained in $\left(0, x_{0}\right)$.

Next, we prove that $\left\{\lambda_{n}\right\}_{n=0}^{\infty},\left\{\gamma_{n}^{-}\right\}_{n=0}^{\infty}$, and $\left\{\gamma_{n}^{+}\right\}_{n=0}^{\infty}$ determine $q(x)$. By (35) and Theorem 3.2 in [21], we see that $\left\{\lambda_{n}\right\}_{n=0}^{\infty},\left\{\gamma_{n}^{-}\right\}_{n=0}^{\infty}$, and $\left\{\gamma_{n}^{+}\right\}_{n=0}^{\infty}$ can uniquely determine $q(x)$, a.e., on $(0,1)$. The proof is therefore complete.

Remark 3. Based on the proof of Theorem 2, we know that two disjoint spectra $\left\{\lambda_{n}\right\}_{n=0}^{\infty}$ and $\left\{\mu_{n}\right\}_{n=0}^{\infty}$ determine $\left\{\gamma_{n}\right\}_{n=0}^{\infty}$ uniquely rather than $\left\{\gamma_{n}^{-}\right\}_{n=0}^{\infty}$ and $\left\{\gamma_{n}^{+}\right\}_{n=0}^{\infty}$. Thus, in order to obtain the uniqueness of $q(x)$, we need to identify $\left\{\gamma_{n}^{-}\right\}_{n=0}^{\infty}$ and $\left\{\gamma_{n}^{+}\right\}_{n=0}^{\infty}$ from $\left\{\gamma_{n}\right\}_{n=0}^{\infty}$. To this end, we have to employ the number of zeros of eigenfunctions, and the condition two spectra are disjoint, if not, we may not guarantee the uniqueness of $q(x)$. For example, if $x_{0}=1 / 2$, then the eigenvalues of $L$ and $L^{ \pm}$have the following asymptotic expressions:

$$
\begin{aligned}
& \sqrt{\lambda_{n}}=\left(n+\frac{1}{2}\right) \pi+\frac{w}{n}+\frac{\kappa_{n}}{n}, \\
& \sqrt{\gamma_{n}^{-}}=(2 n+1) \pi+\frac{w^{-}}{n}+\frac{\kappa_{n}^{-}}{n}, \\
& \sqrt{\gamma_{n}^{+}}=(2 n+2) \pi+\frac{w^{+}}{n}+\frac{\kappa_{n}^{+}}{n},
\end{aligned}
$$

where $\quad w=1 / 2 \int_{0}^{1} q(x) d x, w^{-}=\int_{0}^{1 / 2} q(x) d x, w^{+}=\int_{1 / 2}^{1} q(x) d x$ and $\left\{\kappa_{n}\right\},\left\{\kappa_{n}^{-}\right\},\left\{\kappa_{n}^{+}\right\} \in l_{2}$. It is easy to see that there exists a positive integer $N$ such that, for $n>N$,

$$
\lambda_{2 n}<\gamma_{n}^{-}:=\gamma_{2 n}<\lambda_{2 n+1}<\gamma_{n}^{+}:=\gamma_{2 n+1}<\lambda_{2 n+2},
$$

while we cannot identify $\left\{\gamma_{n}^{-}\right\}_{n=0}^{\infty}$ and $\left\{\gamma_{n}^{+}\right\}_{n=0}^{\infty}$ from $\left\{\gamma_{n}\right\}_{n=0}^{\infty}$ for $n \leq N$, which means that there will be at most a finite number of $q(x)$ corresponding to two spectra $\left\{\gamma_{n}\right\}_{n=0}^{\infty}$ and $\left\{\lambda_{n}\right\}_{n=0}^{\infty}$ in virtue of Theorem 3.2 in [21]. Hence, we employ the number of zeros of eigenfunctions, and the condition two spectra are disjoint to guarantee the uniqueness of $q(x)$. And we have not found an example that the joint spectra determine the potential uniquely.

\section{Data Availability}

No data were used to support this study.

\section{Conflicts of Interest}

The author declares that there are no conflicts of interest regarding the publication of this paper.

\section{Authors' Contributions}

The author conceived of the study, drafted the manuscript, and approved the final manuscript.

\section{Acknowledgments}

The author is very grateful to the handling editors and the reviewers for the careful reading of this manuscript and offering valuable comments for this manuscript.

\section{References}

[1] B. Simon, "Spectral analysis of rank one perturbations and applications," CRM Proceedings and Lecture Notes, vol. 8, pp. 109-149, 1995.

[2] T. Kato, Perturbation Theory for Linear Operators, Springer, 1980.

[3] S. Albeverio, R. O. Hryniv, and L. P. Nizhnik, "Inverse spectral problems for non-local Sturm-Liouville operators," Inverse Problems, vol. 23, pp. 523-535, 2007.

[4] G. Borg, "Eine Umkehrung der Sturm-Liouvilleschen Eigenwertaufgabe," Acta Mathematica, vol. 78, pp. 1-96, 1946. 
[5] R. del Rio and M. Kudryavtsev, "Inverse problems for Jacobi operators: I," Interior mass-spring perturbations in finite systems, Inverse Problems, vol. 28, article 055007, 2012.

[6] R. del Rio, M. Kudryavtsev, and L. O. Silva, "Inverse problems for Jacobi operators IV: interior mass-spring perturbations of semi-infinite systems," Inverse Problems, vol. 33, article $055014,2017$.

[7] O. N. Litvinenko and V. I. Soshnikov, The Theory of Heterogenious Lines and their Applications in Radio Engineering, Radio, Moscow, 1964.

[8] A. D. Wentzell, "On boundary conditions for multidimensional diffusion processes," Teorija Verojatnostei i ee Primenenija, vol. 4, pp. 172-185, 1959.

[9] S. Albeverio and L. P. Nizhnik, "Schrödinger operators with nonlocal point interactions," Journal Mathematical Analysis and Applications, vol. 332, pp. 884-895, 2007.

[10] M. H. Annaby, H. A. Hassan, and O. H. El-Haddad, "Perturbed discrete Sturm-Liouville problems and associated sampling theorems," Rocky Mountain Journal of Mathematics, vol. 39, pp. 1781-1807, 2009.

[11] O. Bourget, V. Cortes, R. del Rio, and C. Fernandez, "Resonances under rank-one perturbations," Journal of Mathematical Physics, vol. 58, article 093502, 2017.

[12] P. Freitas, "A nonlocal Sturm-Liouville eigenvalue problem," Proceedings of the Royal Society of Edinburgh Section A Mathematics, vol. 124, pp. 169-188, 1994.

[13] F. Gesztesy and B. Simon, "Rank one perturbations at infinite coupling," Journal of Functional Analysis, vol. 128, pp. 245252, 1995.

[14] V. V. Kravchenko, Direct and Inverse Sturm-Liouville Problems: A Method of Solution, Frontiers in Mathematics, Birkhauser, Cham, Series, 2020.

[15] L. P. Nizhnik, "Inverse eigenvalue problems for nonlocal Sturm-Liouville operators," Methods of Functional Analysis and Topology, vol. 15, pp. 41-47, 2009.

[16] L. P. Nizhnik, "Inverse nonlocal Sturm-Liouville problem," Inverse Problems, vol. 26, pp. 635-684, 2010.

[17] L. P. Nizhnik, "Inverse eigenvalue problems for nonlocal Sturm-Liouville operators on a star graph," Methods of Functional Analysis and Topology, vol. 18, pp. 68-78, 2012.

[18] X. Wu, P. Niu, and G. Wei, "An inverse eigenvalue problem for a nonlocal Sturm-Liouville operator," Journal of Mathematical Analysis and Applications, vol. 494, p. 124661, 2021.

[19] V. A. Zolotarev, "Direct and inverse problems for an operator with nonlocal potential," Russian Academy of Sciences Sbornik Mathematics, vol. 203, pp. 1785-1807, 2012.

[20] V. A. Zolotarev, "Inverse spectral problem for the operators with non-local potential," Mathe matische Nachrichten, vol. 291, pp. 1502-1523, 2019.

[21] O. Boyko, V. Pivovarchik, and C. F. Yang, "On solvability of three spectra problem," Mathematische Nachrichten, vol. 289, pp. 1727-1738, 2016.

[22] V. N. Pivovarchik, "An inverse Sturm-Liouville problem by three spectra," Integral Equations and Operator Theory, vol. 34, pp. 234-243, 1999.

[23] G. Freiling and V. Yurko, Inverse Sturm-Liouville Problems and their Applications, Nova Science Publishers, 2001.

[24] J. B. Conway, Functions of One Complex Variable, Springer Verlag, 1995.

[25] B. M. Levitan and I. S. Sargsian, Sturm-Liouville and Dirac Operators, Mathematics and its Applications, 1991. 